\title{
Hepatic Extramedullary Hematopoiesis and Macrophages in the Adult Mouse: Histometrical and Immunohistochemical Studies
}

\author{
Yuji Sonoda Kazunobu Sasaki \\ Department of Anatomy, Kawasaki Medical School, Kurashiki, Japan
}

\section{Key Words}

Phenylhydrazine - Central macrophage - Erythropoiesis • Kupffer cell $\cdot$ ER-HR3

\begin{abstract}
Fetal liver hematopoiesis in mice disappears approximately 2 weeks after birth; however, under experimental acute anemia extramedullary hematopoiesis occurs in the livers of adult mice. The hematopoietic foci in the liver during extramedullary hematopoiesis contain erythroblasts and macrophages. In this study, the extent of involvement of macrophages in the development and involutional process of the hematopoietic foci in adult mice livers was clarified by experimentally inducing extramedullary hematopoiesis. Hematopoietic cells appeared in the livers 2 days after phenylhydrazine ( $\mathrm{PHZ}$ ) injections. The number and area of the foci increased rapidly, reaching peak values on the sixth day. F4/80-positive macrophages were observed in the sinusoids as well as the hematopoietic foci, and were tightly surrounded by erythroblasts. Sinusoidal macrophages in normal adult livers were positive for F4/80 but negative for ER-HR3. However, in extramedullary hematopoiesis-induced livers, sinusoidal macrophages became positive for ER-HR3 antibodies. The number of ER-HR3-positive macrophages was $9.2 \pm 2.9 / \mathrm{mm}^{2}$ on the second day after PHZ was administered, and increased to $200.3 \pm 4.2 / \mathrm{mm}^{2}$ on the sixth day. On the seventh day after the PHZ injections, the number decreased and they were no longer detected at 30 days after $\mathrm{PHZ}$ was injected. The present study revealed that erythro-
\end{abstract}

blasts accumulate around sinusoidal macrophages to form an erythroblastic island with a central macrophage similar to erythropoiesis in the fetal liver. Furthermore, in line with development and regression of extramedullary hematopoiesis, macrophages express ER-HR3, a hematopoiesis related antigen.

Copyright $\odot 2012$ S. Karger AG, Basel

\section{Introduction}

During intrauterine life the liver is a major hematopoietic organ and is mainly involved in erythropoiesis. We previously clarified that in terms of the three-dimensional surface structures of the central macrophages of erythroblastic islets in the fetal liver and the adult splenic red pulp, macrophages play important roles in both the formation of the hematopoietic niche and erythroid differ-

\begin{tabular}{ll}
\hline Abbreviations used in this paper \\
\hline $\mathrm{CV}$ & central vein \\
$\mathrm{En}$ & endothelial cell \\
$\mathrm{Er}$ & erythrocyte \\
$\mathrm{H}$ & hepatocyte \\
$\mathrm{Mp}$ & macrophage \\
$\mathrm{PHZ}$ & phenylhydrazine \\
$\mathrm{PV}$ & portal vein \\
$\mathrm{S}$ & sinusoid
\end{tabular}

\begin{tabular}{ll}
\hline KARGER & ( 2012 S. Karger AG, Basel \\
1422-6405/12/1966-0555\$38.00/0 \\
$\begin{array}{l}\text { Fax +41 61 306 1234 } \\
\begin{array}{l}\text { E-Mail karger@karger.ch } \\
\text { www.karger.com }\end{array}\end{array}$ & $\begin{array}{l}\text { Accessible online at: } \\
\text { www.karger.com/cto }\end{array}$
\end{tabular}


entiation. Furthermore, macrophages changed not only the surface morphology but also the cell membrane antigen in response to changes of the erythropoietic activity caused by aging and pregnancy [Sasaki et al., 1981; Sonoda and Sasaki, 2006, 2008]. Fetal hematopoiesis ceases in the liver in mice about 1 week after birth and, under normal physiological conditions, hematopoiesis does not occur in adult mice livers. However, under myelosuppression by various pathological lesions, experimental phenylhydrazine ( $\mathrm{PHZ}$ ) injections or ischemia-inducible acute anemia, extramedullary hematopoiesis can occur in adult mouse livers [Ploemacher and van Soest, 1977; Cardier and Barberá-Guillem, 1997]. Currently, there is no detailed information on the ultrastructure and expression of cell membrane antigens in relation to extramedullary hematopoiesis in adult mice. The purpose of this study was to clarify the involvement of macrophages in the development of and the involutional process of the hematopoietic foci in the livers of splenectomized adult mice by experimentally inducing extramedullary hematopoiesis.

\section{Materials and Methods}

Male ICR mice at 60 days of age were used for this study. A total of 64 mice were used. Extramedullary hematopoiesis in the liver was induced by injecting phenylhydrazine hydrochloride (Sigma, USA) into splenectomized mice. Splenectomy was performed at 60 days of age via a left-flank incision under light sevoflurane anesthesia with antiseptic precautions. From 7 days after the splenectomy, mice were intraperitoneally injected with PHZ at a concentration of $25 \mathrm{mg} / \mathrm{kg}$ in $0.1 \mathrm{ml}$ of phosphate-buffered saline ( $\mathrm{pH}$ 7.6). This was conducted at $24 \mathrm{~h}$ intervals for 3 days. The mice were sacrificed under deep anesthesia using sodium pentobarbital $(50 \mathrm{mg} / \mathrm{kg}$ ) and the livers were removed at $0,2,4,6$, $8,12,17$ and 30 days after the last PHZ injection for light and electron microscopy. More than 5 mice were used in each group. The livers of splenectomized mice that had not been injected with PHZ were used as controls. These experiments were approved by the Animal Research Committee of Kawasaki Medical School (No. 10-022) and were conducted according to the Guide for the Care and Use of Laboratory Animals of Kawasaki Medical School.

\section{Light Microscopic Observations}

The livers were fixed in $4 \%$ paraformaldehyde in $0.1 \mathrm{M}$ phosphate buffer $\left(\mathrm{pH} 7.4,4^{\circ} \mathrm{C}\right)$ for $4 \mathrm{~h}$. They were embedded in paraffin and sectioned $4 \mu \mathrm{m}$ thick for the following histochemical procedures.

\section{Immunohistochemistry}

Two rat anti-mouse macrophage monoclonal antibodies, ERHR3 (Funakoshi, Japan) as a marker of macrophages associated with erythropoiesis and F4/80 (Cosmobio, Japan) as a pan-macrophage marker, were used. The avidin-biotin complex method (Vectastain Elite ABC kit; Vector Laboratories, Burlingame,
Calif., USA) was employed. Sections were treated with $99 \%$ methanol containing $0.3 \% \mathrm{H}_{2} \mathrm{O}_{2}$ for $30 \mathrm{~min}$, and were then immersed in $0.1 \%$ normal rabbit serum in phosphate-buffered saline $(\mathrm{pH}$ 7.6) for $1 \mathrm{~h}$ at $25^{\circ} \mathrm{C}$. These sections were either incubated in $\mathrm{F} 4 / 80$ antibody (dilution 1:300) or ER-HR3 antibody (dilution 1:300) for $2 \mathrm{~h}$ at $25^{\circ} \mathrm{C}$. After incubation in the primary antibodies, the sections were treated with biotinylated anti-rat IgG antibody for 30 $\mathrm{min}$ and then immersed in $\mathrm{ABC}$ reagent for $30 \mathrm{~min}$. For visualization of the immunoreactions, $0.02 \% 3,3^{\prime}$-diaminobenzidine containing $0.005 \%$ hydrogen peroxide and $50 \mathrm{~mm}$ Tris- $\mathrm{HCl}$ buffer ( $\mathrm{pH}$ 7.6) was used. Immunostained sections were counterstained with Mayer hematoxylin. For negative controls, sections were reacted with $0.1 \%$ normal rabbit serum albumin instead of the primary antibodies. In addition to the immunohistochemical observations, adjacent sections were stained with hematoxylin-eosin in order to assess the general architecture of liver hematopoiesis at each time point.

Quantitation of Extramedullary Hematopoiesis in the Livers

Development of extramedullary hematopoiesis in the adult mice livers was histometrically quantified by obtaining the area proportions of hematopoietic compartments as well as the number of ER-HR3 labeled cells. The areas of hematopoietic foci and the numbers of either ER-HR3-positive cells or F4/80-positive cells were measured from digital images of the liver sections using Image J software (version 1.4.4, NIH, USA). The digital images measured $246,400 \mu \mathrm{m}^{2}(560 \mu \mathrm{m} \times 440 \mu \mathrm{m})$, and were obtained by using a $\times 20$ objective. These were then imported into Adobe Photoshop (CS3, Adobe Systems Inc., San Jose, Calif., USA). Five randomly selected digital images of 5 mice were used at each time point.

Profiles of the hematopoietic foci containing more than two hematopoietic cells were traced onto digital images in order to obtain their profile areas. The proportions of the hematopoietic compartment in the liver parenchyma were obtained.

Regarding the number and area of hematopoietic foci, the foci frequencies were obtained at each time point from a total of 1,696 hematopoietic foci. The values obtained were statistically evaluated by Student's t test.

\section{Electron Microscopic Observations}

The removed livers were quickly cut into $1 \mathrm{~mm}^{3}$ blocks and immersed in $2.5 \%$ glutaraldehyde in $0.1 \mathrm{M}$ phosphate buffer for $3 \mathrm{~h}$. Blocks of tissue were then post-fixed with $1 \%$ osmium tetroxide for $2 \mathrm{~h}$ and embedded in Epon 812. Ultrathin sections were cut and mounted onto formvar film-coated single-hole copper grids. After double staining in uranyl acetate and lead citrate, observations were carried out with a JEM-1400 operating at $80 \mathrm{kV}$ or an $\mathrm{H}-7100$ operating at $75 \mathrm{kV}$.

\section{Results}

\section{Development of Extramedullary Hematopoiesis in Livers after $\mathrm{PHZ}$ Injections}

After PHZ injections extramedullary hematopoiesis primarily occurred in the sinusoid of the hepatic lobules. Spherical or ellipsoidal hematopoietic foci of various siz- 


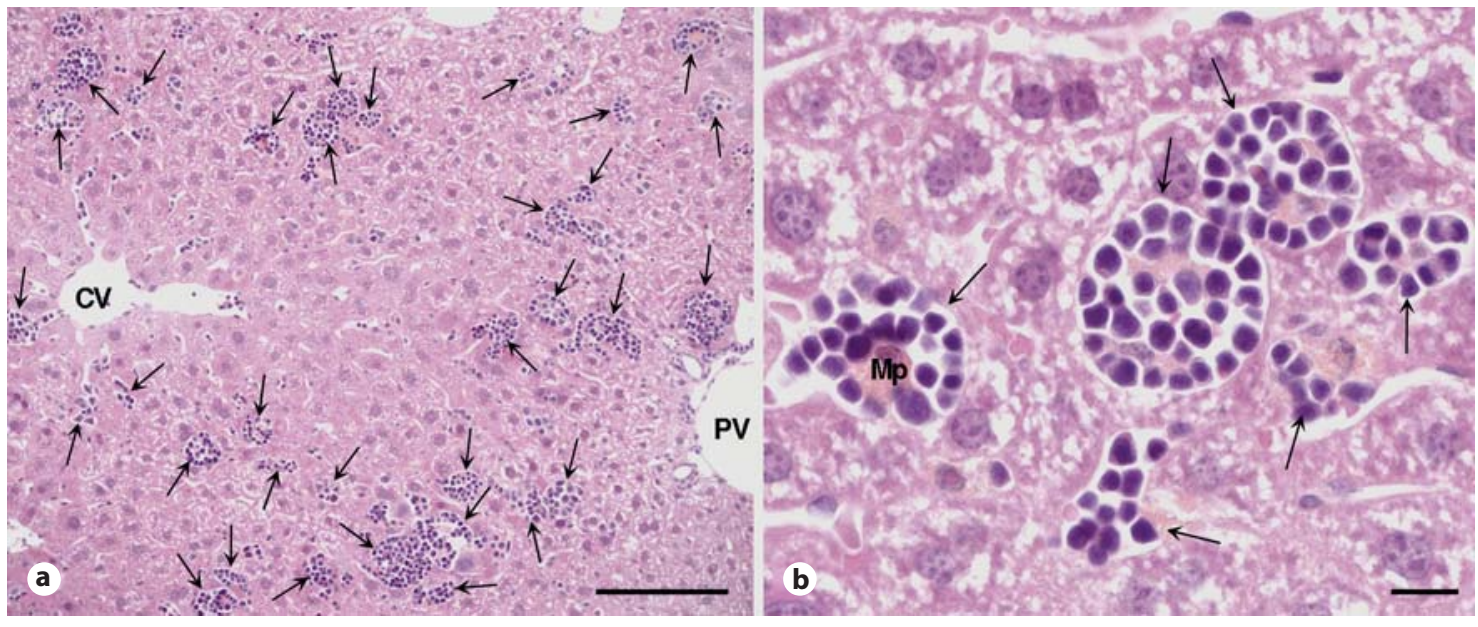

Fig. 1. HE-stained liver after PHZ injection. a Low magnification. Hematopoietic foci (arrows) were observed throughout the liver parenchyma as either spherical or ellipsoidal in shape. They were often situated near interlobular connective tissues containing the portal vein $(\mathrm{PV}) . \mathrm{CV}=$ Central vein. Scale bar $=50 \mu \mathrm{m}$. b High magnification of the hematopoietic foci. The hematopoietic foci (arrows) were mostly spherical in shape, containing numerous erythroblasts and a few macrophages $(\mathrm{Mp})$. Scale bar $=10 \mu \mathrm{m}$.

es were scattered throughout the liver parenchyma and were often situated near interlobular connective tissues (fig. 1a). The hematopoietic foci were mainly erythropoietic, containing numerous erythroblasts and the majority of the foci contained some macrophages (fig. 1b). Megakaryocytes and a few granuloid hematopoietic cells were observed in the hematopoietic foci.

Two days after the last PHZ injection extramedullary hematopoiesis appeared in the liver, and thereafter a marked increase in the number of hematopoietic cells was observed. Figure 2 shows the area of the hematopoietic compartment in livers from 0 to 30 days after the last PHZ injections. The area of the hematopoietic compartment accounted for $0.05 \pm 0.02 \%$ of hepatic parenchyma at 2 days after the injections, $1.63 \pm 0.25 \%$ at 4 days and reached a maximum of $8.25 \pm 2.34 \%$ at 6 days after the last injections. Following the sixth day after the last PHZ injection, a rapid decline in the area of the hematopoietic compartment was observed until 12 days after, when it accounted for $0.22 \pm 0.12 \%$.

Figure 3 shows the distribution of profile area of foci between 2 and 12 days after the last injection. Hematopoietic foci larger than $4,000 \mu \mathrm{m}^{2}$ in profile area were formed at 6 days. At 2 days after the last injection the majority of hematopoietic foci in the profile area measured 50-100 $\mu \mathrm{m}^{2}$, and foci larger than $400 \mu \mathrm{m}^{2}$ appeared after 4 days (fig. 3a, b). Due to the fusion of neighboring hematopoietic foci, large hematopoietic foci $>2,000 \mu \mathrm{m}^{2}$ in

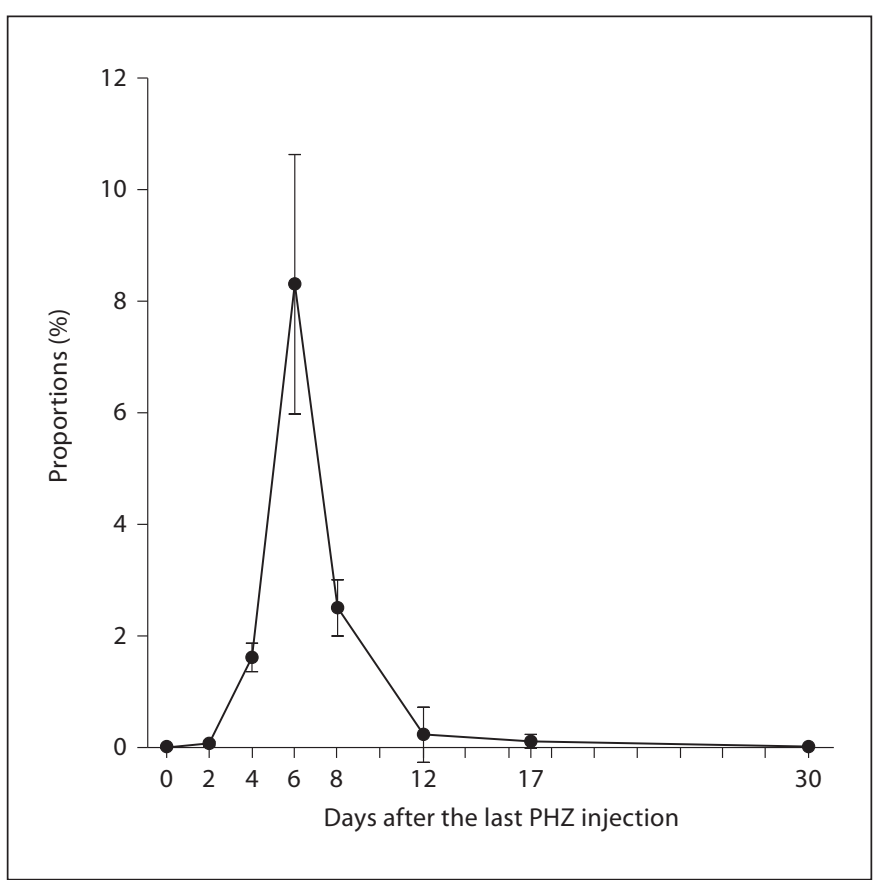

Fig. 2. Area proportions of the hematopoietic compartment in the extramedullary hematopoiesis of the livers after PHZ injections. Mean \pm SD. The proportions showed an increase between 2 and 4 days after the PHZ injections and reached a peak at 6 days after the injections. At 12 days, the proportions became very low, 0.22 $\pm 0.12 \%$. 


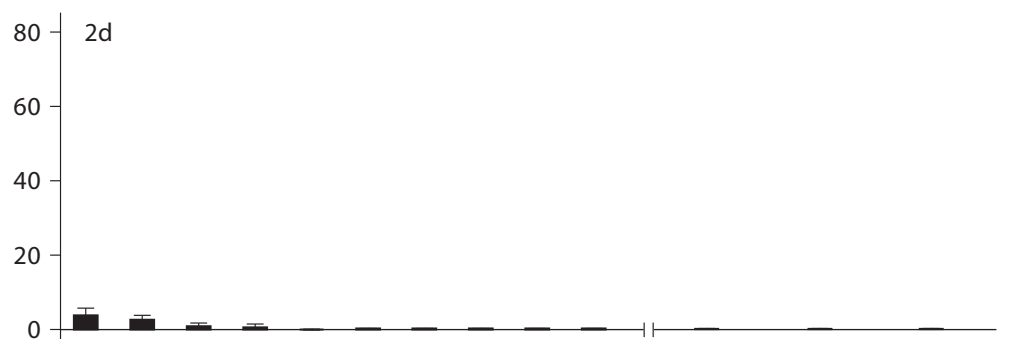

b
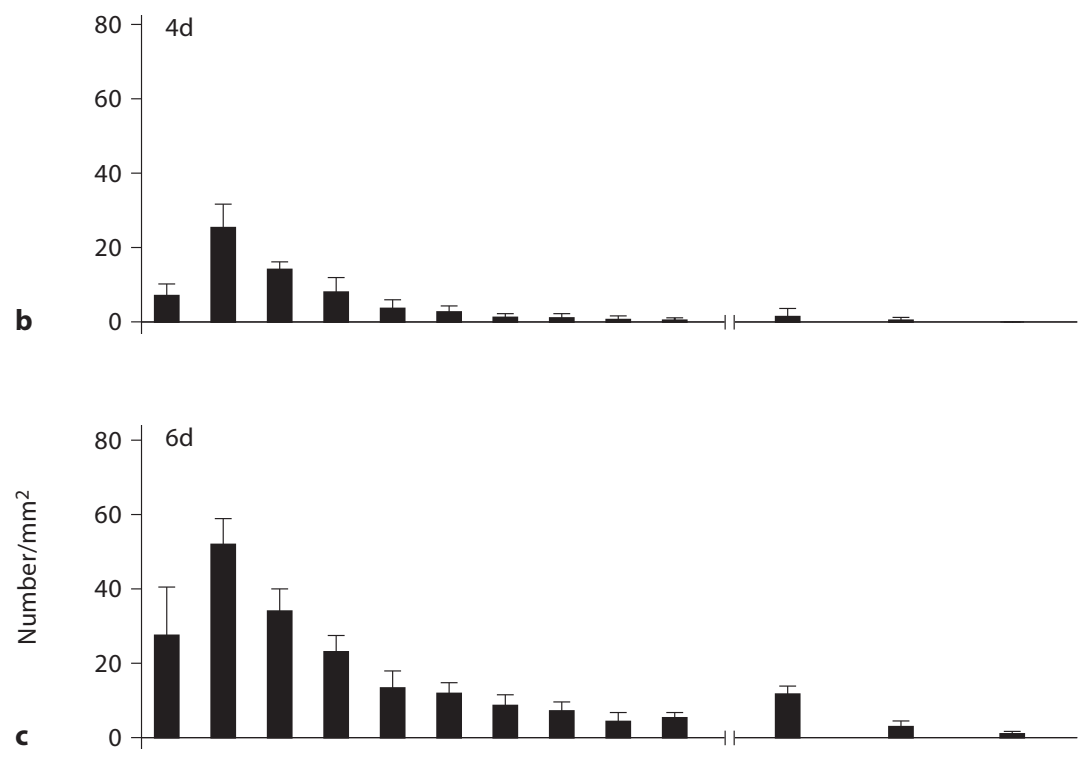

d

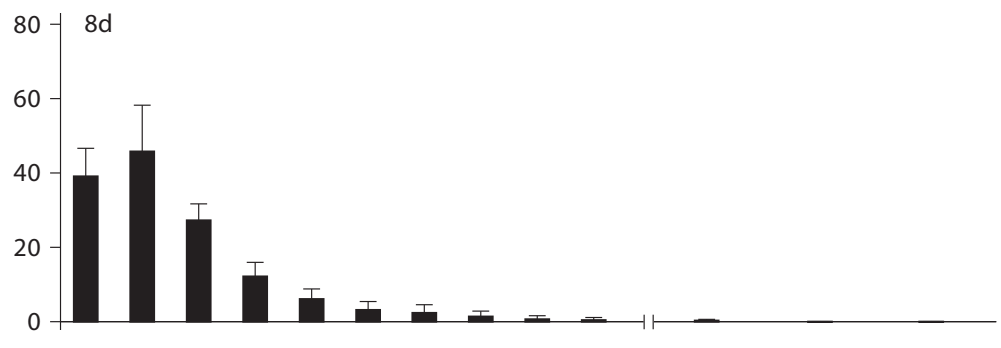

Fig. 3. Size distribution of extramedullary hematopoietic foci. The majority of hematopoietic foci between 4 and 8 days measured $100-200 \mu \mathrm{m}^{2}$ in the profile area. Hematopoietic foci larger than $4,000 \mu^{2}$ appeared at 6 days. At 12 days the hematopoietic foci became very small in size.

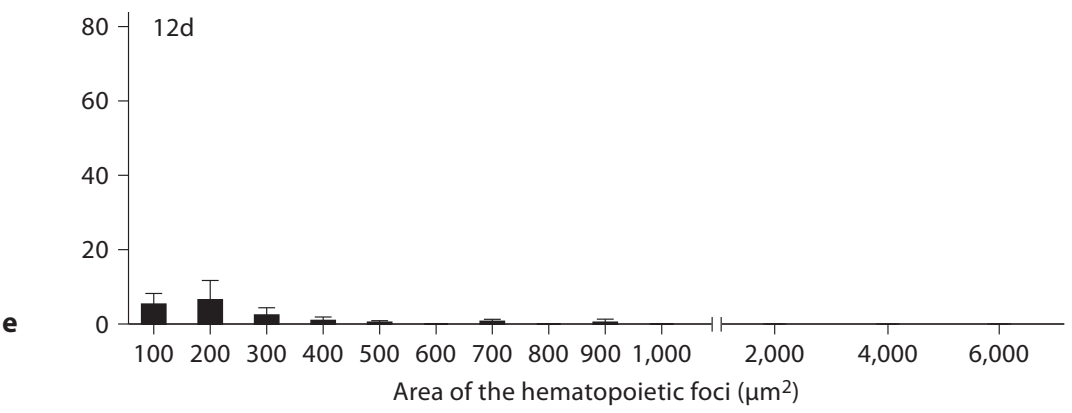




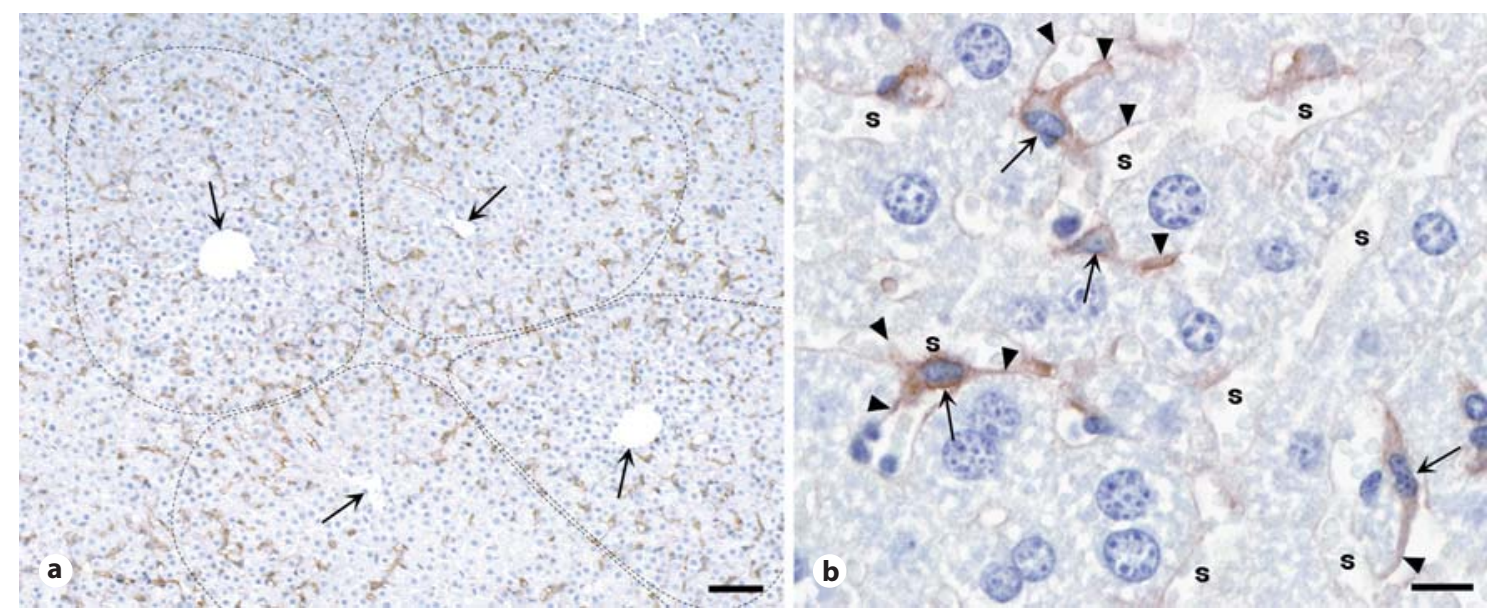

Fig. 4. F4/80-stained liver of a control mouse. a Low-power micrograph. The majority of macrophages were seen at peripheral regions of the hepatic lobules (broken lines) but were rarely observed around the central veins (arrows). Scale bar $=50 \mu \mathrm{m}$. b High-power micrograph. F4/80-positive macrophages (arrows) were irregular, with elongated cell contours, mainly located in the sinusoid (s) and possessed pseudopod-like processes (arrowheads). Scale bar $=10 \mu \mathrm{m}$.

profile area increased in number at 6 days, with the largest area measured at approximately 6,000 $\mu \mathrm{m}^{2}$ (fig. 3c). At 12 days the hematopoietic foci became smaller in size due to a marked depletion of erythroid cells (fig. 3d). This continued until only macrophages remained in the sinusoid as the last cell component of the foci. The morphological unit of liver, the hepatic lobule, is a polygonal structure containing central veins at the center of the lobule. Each lobule was separated by interlobular connective tissues containing the portal triad, portal vein, hepatic artery and bile duct. At 6 days after the last injection, the hematopoietic foci were frequently distributed at the peripheral and intermediate areas of the liver acinus, with low abundance of the foci near to the central vein.

\section{Liver Macrophages during Extramedullary Hematopoiesis \\ Histochemistry}

Normal adult mice livers contained numerous macrophages, which were frequently observed at the peripheral regions of the hepatic lobules (fig. 4a). The macrophages that were located in the sinusoid (fig. $4 \mathrm{~b}$ ) were F4/80-positive but ER-HR3-negative (fig. 5a, c). However, after the PHZ injection the sinusoidal macrophages became ERHR3-positive (fig. 5b, d) and increased in number significantly during the development of extramedullary hematopoiesis. Figure 6 shows numbers of both ER-HR3positive macrophages and F4/80-positive macrophages per hepatic unit area from 0 to 30 days after the last injection. The number of ER-HR3-positive macrophages was $21.9 \pm 7.1 / \mathrm{mm}^{2}$ at 2 days, and then increased rapidly to reach a peak of $431.3 \pm 96.2 / \mathrm{mm}^{2}$ at 6 days, which was approximately 20 -fold greater than the number at 2 days. The peak number of ER-HR3-positive macrophages $\left(431.3 \pm 96.2 / \mathrm{mm}^{2}\right)$ was approximately $60 \%$ of the number of F4/80-positive macrophages. The number of ERHR3-positive macrophages then decreased to $237.8 \pm$ $39.9 / \mathrm{mm}^{2}$ at 8 days, $157.6 \pm 47.0 / \mathrm{mm}^{2}$ at 12 days and 59.2 $\pm 8.9 / \mathrm{mm}^{2}$ at 17 days. Furthermore, all hepatic macrophages became ER-HR3-negative at 30 days. During extramedullary hematopoiesis the mean number of F4/80positive macrophages per hepatic unit area was $759.3 \pm$ $60.4 / \mathrm{mm}^{2}$, and there were no statistically significant changes in the mean numbers during extramedullary hematopoiesis.

At 2 days after the injections, ER-HR3-positive macrophages in the sinusoids were in close contact with the sinusoidal faces of a few hematopoietic cells (fig. 7a), and many of them were surrounded by a single layer of erythroblasts. The macrophages extended long cytoplasmic processes into the surrounding erythroblasts, thereby forming typical erythroblastic islets (fig. 7b). From 4 to 8 days after the injection, ER-HR3-positive macrophages were surrounded by two or more layers of erythroblasts (fig. 7c). Due to the fusion of adjacent hematopoietic foci, numerous ER-HR3-positive macrophages could be ob- 


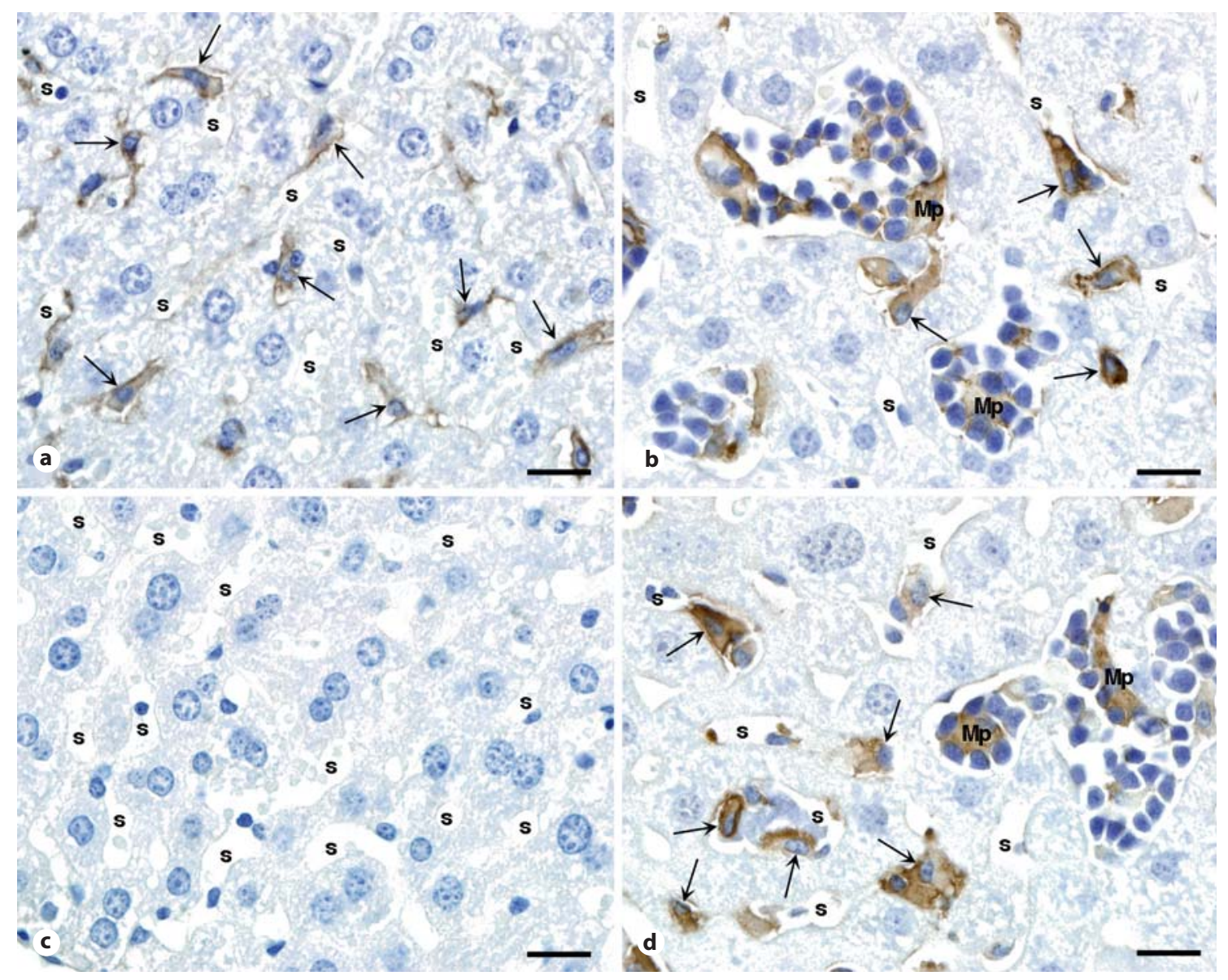

Fig. 5. ER-HR3- and F4/80-stained livers after the PHZ injections. $S=$ Sinusoid. Scale bar $=10 \mu \mathrm{m}$. a F4/80stained control liver. Sinusoidal macrophages (arrows) were F4/80-positive and no hematopoietic foci were observed. b F4/80-stained liver at 6 days after the injection. Numerous hematopoietic foci were observed. Both sinusoidal macrophages (arrows) and macrophages (Mp) in hematopoietic foci were F4/80-positive. c ER-HR3stained control liver. All sinusoidal macrophages were ER-HR3-negative. d ER-HR3-stained liver at 6 days after the injection. Hematopoietic foci contained ER-HR3-positive Mp and the majority of the sinusoidal macrophages (arrows) also became ER-HR-positive.

served in a single large hematopoietic focus (fig. 7d). During extramedullary hematopoiesis sinusoidal macrophages and central macrophages in erythroblastic islets were ER-HR3-positive.

\section{Ultrastructure}

In the control livers, sinusoidal macrophages contained a few inclusions, which were approximately $1 \mu \mathrm{m}$ in diameter. They also contained numerous lysosomes which were measured at $100-200 \mathrm{~nm}$ in diameter. After the PHZ injections, numerous degenerative erythrocytes were observed in the sinusoidal space. Although the cytoplasm of normal erythrocytes generally ap- peared homogeneous, the erythrocytes possessed patchy cytoplasms which contained electron-dense areas with irregular contours and small erythroid fragments approximately $3 \mu \mathrm{m}$ in diameter after the PHZ injections. These were also observed in the sinusoid (fig. 8a). After the PHZ injections, the sinusoidal macrophages actively engulfed both degenerative erythrocytes and small erythroid fragments. They also possessed numerous large inclusions, siderosomes, and had irregular contours in their cytoplasms (fig. 8b). The central macrophages of erythroblastic islets which extended their long, flattened or filamentous cytoplasmic processes among surrounding erythroblasts also contained numerous 


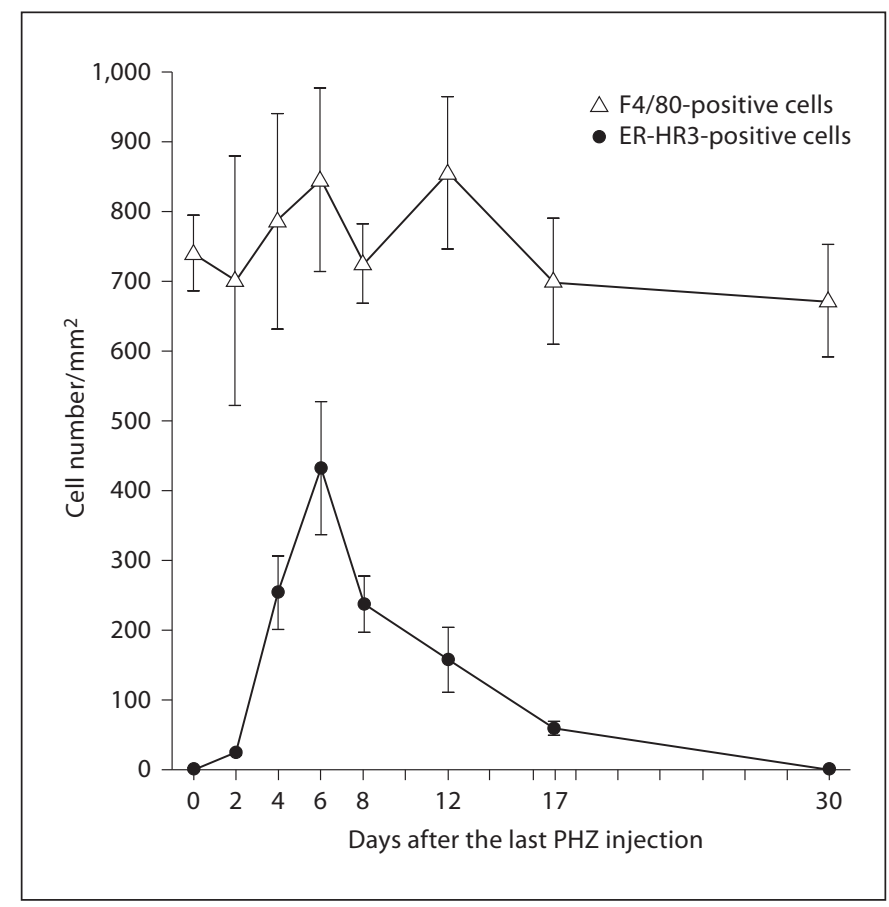

Fig. 6. Number of ER-HR3 - and F4/80-positive cells after the PHZ injection. The number of ER-HR3-positive macrophages showed a marked increase between 2 and 6 days after the PHZ injection. After 6 days, the numbers gradually decreased and all hepatic macrophages became ER-HR3-negative at 30 days. The number of F4/80-positive macrophages was almost constant in the extramedullary hematopoiesis.

large siderosomes (fig. 8c, d). On the central macrophages surface, cell sockets for ery throblasts were formed by cell projections. Erythroblastic islets located in the sinusoidal space were enclosed by thin endothelial cells of the sinusoids.

\section{Discussion}

In the livers of splenectomized adult mice, injections of PHZ induced extramedullary hematopoiesis and the hematopoietic foci consisted of typical erythroblastic islets. After PHZ injections, the cellular constituents of the hematopoietic foci of extramedullary hematopoiesis in the adult livers were similar to those in fetal liver hematopoiesis. This study revealed that erythroblasts accumulate around sinusoidal macrophages to form an erythroblastic island with a central macrophage similar to erythropoiesis in the fetal liver. Furthermore, in line with development and regression of extramedullary hemato-

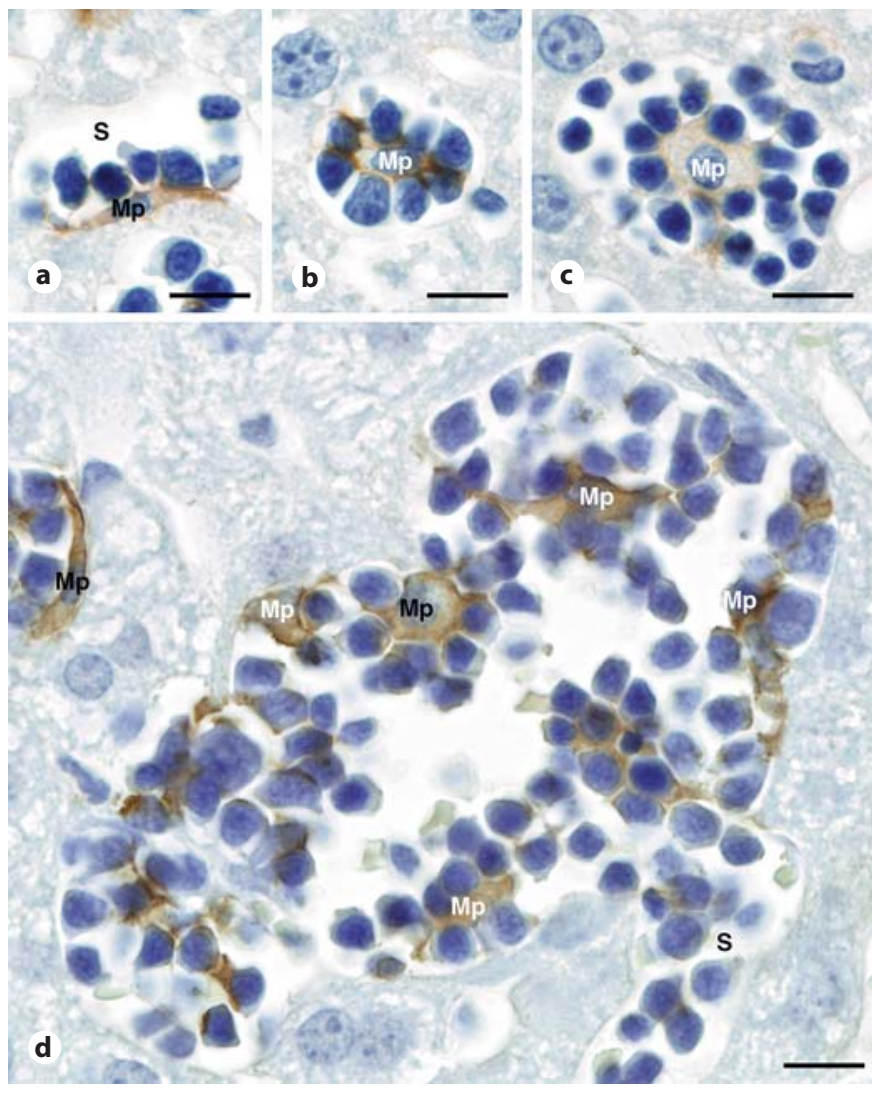

Fig. 7. ER-HR3-positive macrophages in extramedullary hematopoietic foci. $S$ = Sinusoid. Scale bar $=10 \mu \mathrm{m}$. a An ER-HR3-positive sinusoidal macrophage $(\mathrm{Mp})$ is in contact with the sinusoidal face of a few hematopoietic cells at 2 days after the injection. $\mathbf{b}$ A small erythroblastic islet with an ER-HR3-positive Mp at 2 days after the injection. The macrophage in the erythroblastic islet is surrounded by a single layer of erythroblasts. c A large typical erythroblastic islet with an ER-HR3-positive Mp at 6 days after the injection. The central macrophage is surrounded by two or three layers of erythroblasts. d Numerous erythroblastic islets with ER-HR3-positive Mp in a single large hematopoietic focus at 6 days after the injection.

poiesis, macrophages express ER-HR3, a hematopoiesis related antigen.

$\mathrm{PHZ}$ is known to react with both hemoglobin and cytochrome P450 within the erythrocytes to cause severe hemolytic anemia [Di Cola et al., 1988; Maples et al., 1988; Di Cola et al, 1989] and, by experimental injection of PHZ, extramedullary hematopoiesis was induced in murine livers [Ploemacher and van Soest, 1977; BarberáGuillem et al., 1989; Lenox et al., 2009]. Furthermore, the fetal liver following the yolk sac is also known to be a major hematopoietic organ during intrauterine life. After birth, the hepatic hematopoiesis rapidly disappears 

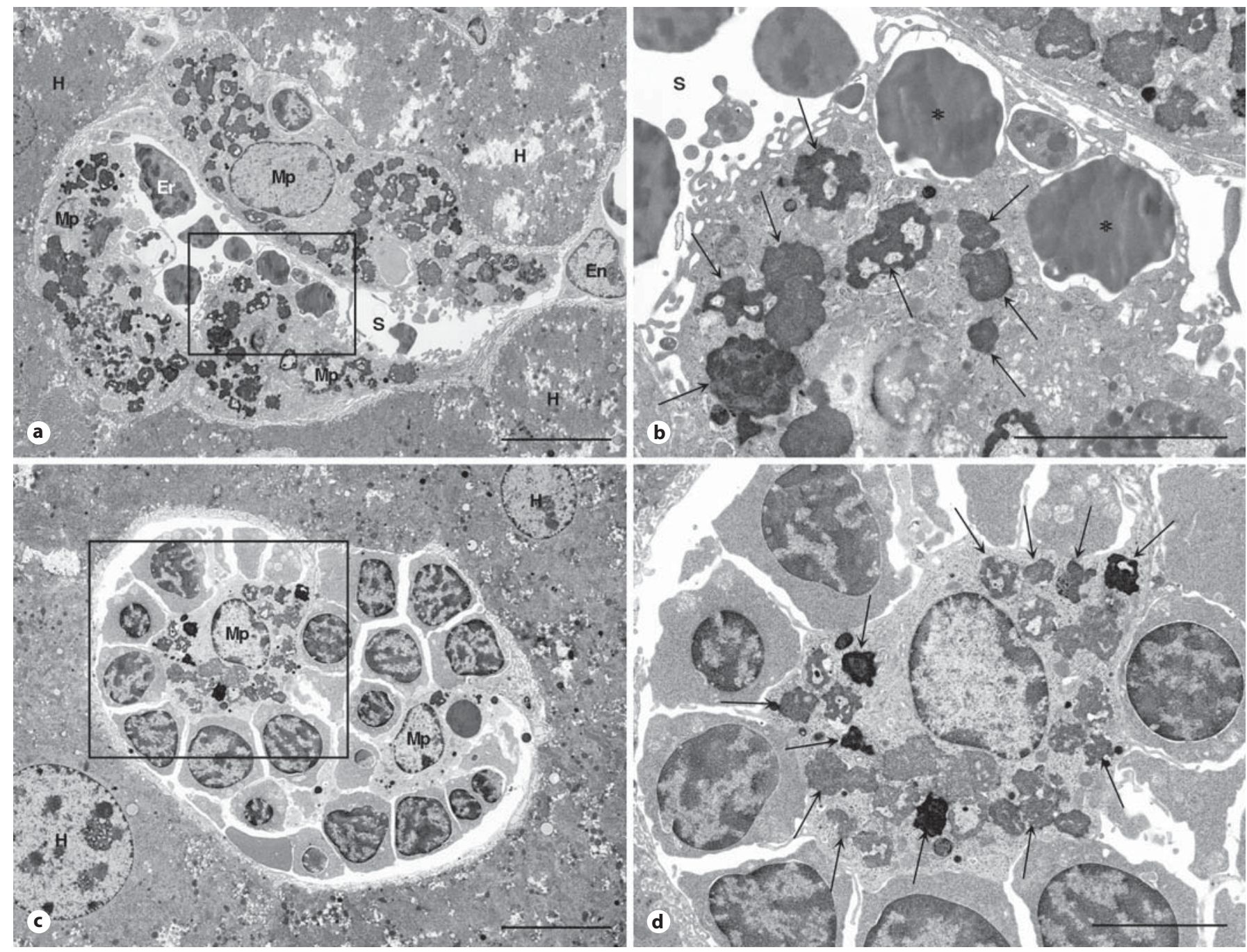

Fig. 8. Ultrastructure of macrophages during extramedullary hematopoiesis. a Low-power micrograph of sinusoidal macrophages at 4 days after the PHZ injection. The cytoplasm expands due to inclusions of varied sizes. En = Endothelial cell; Er = erythrocyte; $\mathrm{H}=$ hepatocyte; $\mathrm{S}=$ sinusoid. Scale bar $=10 \mu \mathrm{m}$. b High-power micrograph of the area shown by the rectangle in a. The macrophages possess numerous inclusions, siderosomes (arrows), derived from erythroid fragments $\left(^{*}\right)$ in their

[Tavassoli, 1991]. However, by injecting PHZ into splenectomized mice extramedullary hematopoiesis reoccurs in adult mice livers. Concerning the hematopoietic foci, PHZ-induced extramedullary hematopoiesis differed from fetal hepatic hematopoiesis in two ways - in the location and distribution of the hepatic lobules. In the fetal liver, the hematopoietic foci occurred in expanded perisinusoidal spaces between hepatocytes and sinusoi-

dal endothelium [Sasaki and Sonoda, 2000]. In contrast, the hematopoietic foci induced by $\mathrm{PHZ}$ injections were found in the sinusoidal lumen and were surrounded by sinusoidal endothelium. In the hematopoietic niche of the fetal livers, EPCR-positive hematopoietic stem cells which possess long-term repopulating ability are known to localize near the Lyve-1-positive sinusoidal endothelium [Iwasaki et al., 2010]. Furthermore, during the de- 
velopmental process from fetal to adult life, the expression of membrane phenotype of the sinusoidal endothelium changes from CD34-positive and $\mathrm{Fc} \gamma \mathrm{R}$-negative in the fetus, to CD34-negative and FcyR-positive in the adult [Nonaka et al., 2007]. Our previous study showed that the sinusoidal endothelium possesses numerous large pores allowing hematopoietic cells to easily move into perivascular spaces at the peak of fetal liver hematopoiesis. However, these large pores disappeared shortly after birth [Wang et al., 1998]. A developmental change was observed in the hepatic progenitor cell type, from Sox9-negative to Sox9-positive progenitors during the functional change of the liver in the middle embryonic stage [Furuyama et al., 2011]. Miyajima et al. [2000] reported that due to the differentiation of fetal hepatic cells, mature hepatic cells lose the ability to support hematopoiesis. In light of these considerations, the difference in localization of the hematopoietic foci between fetal liver hematopoiesis and extramedullary hematopoiesis after the PHZ injections might be closely related to the developmental changes of cellular characterization of both hepatocytes and sinusoidal endothelium.

After the PHZ injections, the majority of hematopoietic foci were distributed near the interlobular connective tissues, with low abundance of the foci near to the central vein. In contrast, hematopoietic foci in fetal livers are equally distributed throughout the hepatic lobules [Sasaki and Sonoda, 2000]. Concerning the localization of the hematopoietic foci in the extramedullary hematopoiesis of the liver after PHZ has been injected, BarberáGuillem et al. [1989] reported that the distribution of hematopoietic foci within liver acini differed between postnatal and phenylhydrazine-treated adult mice, and Lenox et al. [2009] reported that the expression of BMP-4, induced by acute anemia due to hypoxia, was closely interrelated to the localization of hematopoietic foci. Also, Cardier and Barberá-Guillem [1997] reported that LEC type-1 of the hepatic periportal sinusoid was important in the hematopoietic microenvironment for supporting the proliferation and differentiation of hematopoietic cells. Livers contain numerous macrophages and approximately $80 \%$ of the total macrophages in the body accumulate in the liver [Ross and Auger, 2002]. We found erythroblasts clustered around the sinusoidal macrophages. In adult livers, sinusoidal macrophages are distributed primarily in the peripheral region of hepatic lobules [Naito et al., 1990]. As shown in figure 4a, the same results were obtained in the F4/80-immunostained sections. Therefore, the distribution pattern of hematopoietic foci in the hepatic lobules was considered to reflect sinusoidal macrophage distribution, in addition to both LEC type-1 distribution and the expression of BMP-4 in lobules. The ER-HR3 expression of macrophages showed a marked change in response to erythropoietic activity in the murine spleen [Sonoda and Sasaki, 2008] and in extramedullary hematopoiesis of the liver.

Naito et al. [1997] reported that on the basis of endogenous peroxidase activity, central macrophages of erythroblastic islets in murine fetal livers were different to sinusoidal macrophages in neonates. The VCAM-1 expression on Kupffer cells showed a significant change in response to the extramedullary erythropoiesis induced by nitrogen-containing bisphosphonate in splenectomized mice, and Kupffer cells could change their phenotype to function as stromal cells for erythropoiesis [Otsuka et al., 2011]. We found ER-HR-negative sinusoidal macrophages became ER-HR-positive during the development of the extramedullary hematopoiesis, in order to become the central macrophages of erythroblastic islets. The cytoplasm of the central macrophage was also found to contain numerous inclusions derived from erythrocyte destruction. Therefore, similar to fetal macrophages in embryonic livers, hepatic sinusoidal macrophages could become hematopoietic again after injections of PHZ.

The ER-HR3 antigen is known to be expressed on macrophages associated with erythropoiesis in the bone marrow and spleen [de Jong et al., 1994]; however, the functional significance of ER-HR3 has not been fully elucidated. The ER-HR3 antigen appeared to reflect the changes of erythropoietic activity of the macrophages in the liver during extramedullary hematopoiesis. In addition, erythroblasts almost disappeared from the livers at 12 days after PHZ was injected, whilst numerous ERHR3-positive macrophages remained. At the final stage of extramedullary hematopoiesis of the liver, a difference was observed between the number of hematopoietic cells and the expression pattern of ER-HR3 on macrophages. Therefore, in comparison to hematopoietic cells, macrophages clearly displayed evidence of extraembryonic erythropoiesis after PHZ was injected.

\section{Acknowledgement}

This work was supported in part by a research project grant from Kawasaki Medical School (No. 22-A34, 2011). 


\section{References}

-Barberá-Guillem, E., R. Ayala, F. Vidal-Vanaclocha (1989) Differential location of hemopoietic colonies within liver acini of postnatal and phenylhydrazine-treated adult mice. Hepatology 9: 29-36.

Cardier, J.E., E. Barberá-Guillem (1997) Extramedullary hematopoiesis in the adult mouse liver is associated with specific hepatic sinusoidal endothelial cells. Hepatology 26: 165 175.

$\checkmark$ de Jong, J.P., J.S. Voerman, A.J. van der SluijsGelling, R. Willemsen, R.E. Ploemacher (1994) A monoclonal antibody (ER-HR3) against murine macrophages. I. Ontogeny, distribution and enzyme histochemical characterization of ER-HR3-positive cells. Cell Tissue Res 275: 567-576.

Di Cola, D., P. Battista, S. Santarone, P. Sacchetta (1989) Fragmentation of human hemoglobin by oxidative stress produced by phenylhydrazine. Free Radic Res Commun 6: 379386.

Di Cola, D., P. Sacchetta, P. Battista (1988) Proteolysis in human erythrocytes is triggered only by selected oxidative stressing agents. Ital J Biochem 37: 129-138.

Furuyama, K., Y. Kawaguchi, H. Akiyama, M. Horiguchi, S. Kodama, T. Kuhara, S. Hosokawa, A. Elbahrawy, T. Soeda, M. Koizumi, T. Masui, M. Kawaguchi, K. Takaori, R. Doi, E. Nishi, R. Kakinoki, J.M. Deng, R.R. Behringer, T. Nakamura, S. Uemoto (2011) Continuous cell supply from a Sox9-expressing progenitor zone in adult liver, exocrine pancreas and intestine. Nat Genet 43: 34-41.
Iwasaki, H., F. Arai, Y. Kubota, M. Dahl, T. Suda (2010) Endothelial protein C receptor-expressing hematopoietic stem cells reside in the perisinusoidal niche in fetal liver. Blood 116: 544-553.

Lenox, L.E., L. Shi, S. Hegde, R.F. Paulson (2009) Extramedullary erythropoiesis in the adult liver requires BMP-4/Smad5-dependent signaling. Exp Hematol 37: 549-558.

Maples, K.R., S.J. Jordan, R.P. Mason (1988) In vivo rat hemoglobin thiyl free radical formation following phenylhydrazine administration. Mol Pharmacol 33: 344-350.

Miyajima, A., T. Kinoshita, M. Tanaka, A. Kamiya, Y. Mukouyama, T. Hara (2000) Role of Oncostatin $\mathrm{M}$ in hematopoiesis and liver development. Cytokine Growth Factor Rev 11: 177-183.

Naito, M., G. Hasegawa, K. Takahashi (1997) Development, differentiation, and maturation of Kupffer cells. Microsc Res Tech 39: 350 364.

Naito, M., K. Takahashi, S. Nishikawa (1990) Development, differentiation, and maturation of macrophages in the fetal mouse liver. J Leukoc Biol 48: 27-37.

Nonaka, H., M. Tanaka, K. Suzuki, A. Miyajima (2007) Development of murine hepatic sinusoidal endothelial cells characterized by the expression of hyaluronan receptors. Dev Dyn 236: 2258-2267.

Otsuka, H., H. Yagi, Y. Endo, N. Nonaka, M. Nakamura (2011) Kupffer cells support extramedullary erythropoiesis induced by nitrogen-containing bisphosphonate in splenectomized mice. Cell Immunol 271: 197-204.
Ploemacher, R.E., P.L. van Soest (1977) Morphological investigation on phenylhydrazine-induced erythropoiesis in the adult mouse liver. Cell Tissue Res 178: 435-461.

Ross, J.A., M.J. Auger (2002) The biology of the macrophage; in Burke B., Lewis C.E. (eds): The Macrophage, ed 2. Oxford: Oxford University Press.

Sasaki, K., G. Matsumura, T. Ito (1981) Effects of pregnancy on erythropoiesis in the splenic red pulp of the mouse: a quantitative electron microscopic study. Arch Histol Jap 44: 429438 .

Sasaki, K., Y. Sonoda (2000) Histometrical and three-dimensional analyses of liver hematopoiesis in the mouse embryo. Arch Histol Cytol 63: 137-146.

Sonoda, Y., K. Sasaki (2006) Three-dimensional surface structure of macrophages in fetal and adult mouse liver: an immunohistochemical light microscopic study. Cells Tissues Organs 184: 166-171.

Sonoda, Y., K. Sasaki (2008) Surface morphology of the central macrophages of erythroblastic islets in the spleen of aged and pregnant mice: an immunohistochemical light microscopic study. Arch Histol Cytol 71: 155-161.

Tavassoli, M. (1991) Embryonic and fetal hemopoiesis: an overview. Blood Cells 17: 269286.

Wang, X.Q., Y. Sonoda, M. Suda, A. Shindo, H. Iwatsuki (1998) Scanning and transmission electron microscopic studies on sinusoidal endothelium in the embryonic, neonatal and adult mouse liver. Kawasaki Med J 24: 69-77. 